University of Nebraska - Lincoln

DigitalCommons@University of Nebraska - Lincoln

Faculty Publications from the Department of Electrical \& Computer Engineering, Department Electrical and Computer Engineering

2015

\title{
Theoretical and Experimental Investigation of an Intensity- Demodulated Fiber-Ring-Laser Ultrasonic Sensor System
}

\author{
G. Liu \\ University of Nebraska-Lincoln, guigenliu@hotmail.com \\ Elliott Sandfort \\ University of Nebraska-Lincoln \\ Lingling $\mathrm{Hu}$ \\ University of Nebraska-Lincoln \\ Tongqing Liu \\ University of Nebraska-Lincoln \\ Ming Han \\ University of Nebraska-Lincoln, mhan@egr.msu.edu
}

Follow this and additional works at: https://digitalcommons.unl.edu/electricalengineeringfacpub

Part of the Computer Engineering Commons, and the Electrical and Computer Engineering Commons

Liu, G.; Sandfort, Elliott; Hu, Lingling; Liu, Tongqing; and Han, Ming, "Theoretical and Experimental Investigation of an Intensity-Demodulated Fiber-Ring-Laser Ultrasonic Sensor System" (2015). Faculty Publications from the Department of Electrical and Computer Engineering. 290.

https://digitalcommons.unl.edu/electricalengineeringfacpub/290

This Article is brought to you for free and open access by the Electrical \& Computer Engineering, Department of at DigitalCommons@University of Nebraska - Lincoln. It has been accepted for inclusion in Faculty Publications from the Department of Electrical and Computer Engineering by an authorized administrator of DigitalCommons@University of Nebraska - Lincoln. 


\title{
Theoretical and Experimental Investigation of an Intensity-Demodulated Fiber-Ring-Laser Ultrasonic Sensor System
}

\author{
Guigen Liu, Elliot Sandfort, Lingling Hu, Tongqing Liu, and Ming Han, Member, IEEE
}

\begin{abstract}
We theoretically and experimentally investigate the performance of an ultrasound detection system based on a fiber ring laser (FRL) whose cavity includes a pair of fiber Bragg gratings. The ultrasonic detection is achieved by the FRL power variations in response to the ultrasound-induced cold-cavity loss modulation of the FRL. The effects of key FRL parameters, including pump power, laser cold-cavity loss, and laser cavity length, on the system signal-to-noise ratio (SNR) performance have been investigated. It is found that the maximum SNR is achieved when the frequency of the ultrasound is the same as that of the FRL relaxation oscillation (RO). Harmonic generations are more prominent when the ultrasonic frequency is at the RO frequency and highly dependent on the strength of the ultrasonic signal. The analysis provides a useful tool for the understanding and optimization of such ultrasonic sensor systems.
\end{abstract}

Index Terms-Fiber-optic sensors, fiber lasers, fiber Bragg gratings, nondestructive testing.

\section{INTRODUCTION}

D RIVEN by the growing desires for the inspection of structural health of sophisticated structures, surveillance of natural disasters (e.g. seismic activities), leak detection of gas and liquid pipelines, etc., ultrasonic sensors based on fiber optics have gained much attention and marvelous progress over the last few decades [1]. Fiber-optic ultrasonic sensors can outperform their counterparts in terms of sensitivity, fabrication cost, ease of deployment, and immunity to electromagnetic interference. To date, various ultrasonic sensor structures including fused-taper optical fiber coupler [2], fiber Bragg gratings (FBGs) [3], [4], fiber interferometers [5], [6], in-line fiber microcantilever [7], and nonadiabatic fiber taper [8], have

Manuscript received September 28, 2014; revised November 20, 2014; accepted November 26, 2014. Date of publication December 4, 2014; date of current version March 27, 2015. This work was supported by the U.S. Office of Naval Research under Grant N000141310159 and Grant N000141410139. The associate editor coordinating the review of this paper and approving it for publication was Prof. Zeljko Ignjatovic.

G. Liu, L. Hu, and M. Han are with the Department of Electrical and Computer Engineering, University of Nebraska-Lincoln, Lincoln, NE 68588 USA (e-mail: guigenliu@hotmail.com; hulinglingdl@gmail.com; mhan3@unl.edu).

E. Sandfort was with the Department of Electrical and Computer Engineering, University of Nebraska-Lincoln, Lincoln, NE 68588 USA. He is now with the Department of Computer Engineering, University of Nebraska-Lincoln, Lincoln, NE 68588 USA (e-mail: etsandfort@gmail.com).

T. Liu was with the Department of Electrical and Computer Engineering, University of Nebraska-Lincoln, Lincoln, NE 68588 USA (e-mail: tongqing@huskers.unl.edu).

Color versions of one or more of the figures in this paper are available online at http://ieeexplore.ieee.org.

Digital Object Identifier 10.1109/JSEN.2014.2378536 been studied. These fiber-optic acoustic/ultrasonic sensors have shown great promise toward practical applications.

Recently, fiber-ring lasers (FRLs) have been developed successfully as a new ultrasonic sensing platform [9]. By incorporating an FBG as the sensing element, the intensity of the FRL output is modulated by the variation of the FBG wavelength due to the wavelength-dependent gain of the erbium doped fiber (EDF). However, the wavelength dependence of the gain medium is weak and difficult to be tuned, significantly limiting the ultrasonic detection sensitivity. To overcome this problem, a narrow tunable optical bandpass filter (TOBPF), whose wavelength is tuned to be on the slope of the reflection spectrum of an FBG, is added into the laser cavity [10]. The FRL lasing wavelength is determined by the TOBPF and its cold-cavity loss is modulated by the ultrasonically-induced spectral shift of the FBG. The ultrasonic signals can be detected by the intensity variations of the FRL in response to the cold cavity loss modulation. Due to the large FBG spectral slope, the detection sensitivity can be significantly improved. Most recently, the dynamic properties of a FRL where one phase-shifted FBG and one apodized FBG are incorporated in the cavity for ultrasonic sensing was investigated by $\mathrm{Wu}$ et al. [11]. In their report, they examined the dynamic response of the FRL to ultrasonic waves in both continuous and burst modes and found that the response of the FRL sensor system has an ultrasound frequency dependence which follows the Lorentz function with the maximum response located at the frequency of the relaxation oscillation (RO). However, its noise performance has not been considered. Rather than the parameter of responsivity, signal-to-noise ratio (SNR) is usually of more interest in practical applications for the characterization of a sensor system, which has not been studied for the FRL ultrasonic systems. In addition, the effects of key FRL parameters, such as pump power, laser cold cavity loss, and laser cavity length, on the system SNR performance which is important for the optimization of such a sensor system, have not been studied.

In this paper, we theoretically and experimentally investigate the effect of these parameters on the performance of a novel FRL ultrasonic sensor system proposed and demonstrated recently by our group [12]. The system achieves the unique capability of self-adaptive operation to large quasistatic background strain perturbations by a laser cavity design that includes a pair of closely-located FBGs engineered to have differential ultrasonic responses. Our studies have led 
(a)

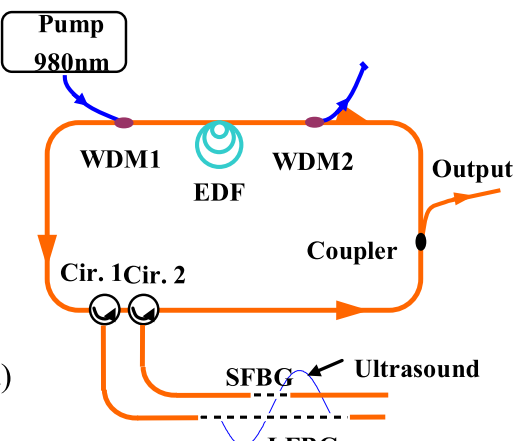

LFBG

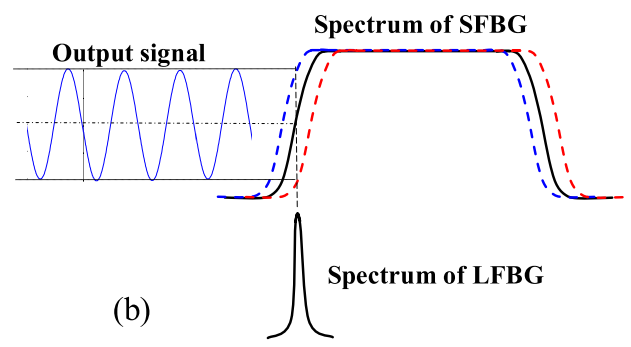

Fig. 1. (a) The FRL ultrasonic sensor system and (b) the reflection spectra of the tandem FBGs.

to several conclusions: (1) the maximum SNR is obtained when the ultrasound frequency is identical to that of RO of the FRL; (2) for large ultrasonic input, the system output contains significant and undesirable harmonic components when the ultrasonic frequency is at or close to the RO frequency; (3) excessive cold cavity loss can significantly reduce the detection sensitivity and SNR of the system, limiting its multiplexing capability; and (4) increased FRL cavity length may also significantly reduce the system detection sensitivity and SNR, which may limit the spatial range of the system.

\section{NUMERICAL ANALYSIS}

\section{A. System Description and Mathematical Model}

The FRL ultrasonic sensor system studied here is schematically shown in Fig. 1(a) and is described in detail in Ref. [12]. Briefly, a section of EDF is pumped by a $980 \mathrm{~nm}$ laser diode through two wavelength division multiplexers (WDMs). The FRL cavity includes a pair of FBGs that are installed sideby-side on a structure. One of the FBGs is strong and short, leading to a broad reflection spectrum with sharp spectral slopes; the other FBG is weak and long, leading to a narrow reflection spectrum that determines the lasing wavelength to be on a spectral slope of the short FBG (SFBG), as schematically shown in Fig. 1(b). It is designed so that the length of the LFBG is comparable to or longer than the ultrasonic wavelength but the length of the SFBG is much shorter than the ultrasonic wavelength, as schematically exhibited in Fig. 1(a). Therefore, the ultrasonic waves impinging onto the FBGs from the axial direction introduce both compressive and tensile strains over the length of the LFBG but much more uniform strains over the SFBG at a given moment. Due to this aperture effect, the ultrasonically-induced FBG spectral shift is much larger for the SFBG than for the LFBG. The differential response to ultrasonic waves results in relative spectral shifts of the FBGs that modulate the FRL cold cavity loss. The laser output power, tapped out of the FRL through a coupler, varies in response to the cavity loss modulation and can be used as the signal for ultrasonic detection. On the other hand, quasi-static background strains only produce identical wavelength shifts of both FBGs and no modulation to the cold cavity loss. Consequently, the system does not respond to the quasi-static background strain.

We have previously developed a model [10] to simulate a FRL sensor based on a FBG and a tunable optical filter where the EDF is described by a two-level model [13]-[15]. Such model is applied to simulate the operation of the FRL sensor system studied here. For convenience, a brief description of the models is provided with small modifications made to the expression of ultrasound-induced cold-cavity cavity loss.

In this model, the longitudinal modes of the FRL are divided into equally-spaced $G$ groups, each of which contains $m$ longitudinal modes of identical parameters. For reasonable simplification, we use the length-averaged inversion level of the upper energy level $N_{2}$, defined as the fraction of atoms in the excited state, whose time-rate change is given by the following time-domain rate equation:

$$
\begin{aligned}
(\rho S l) \frac{d N_{2}}{d t}= & P_{p}\left(1-e^{g_{p} l}\right)-\frac{\rho S l N_{2}}{\tau_{0}}-\sum_{g=1}^{G} \frac{M_{g}}{\tau}\left(1-e^{-g_{g} l}\right) \\
& -\sum_{g=1}^{G} \frac{4 m \gamma_{g} l N_{2}}{\tau}\left(\frac{e^{g_{g} l}-1}{g_{g} l}-1\right)
\end{aligned}
$$

where $t$ denotes time, $P_{p}$ is the pump power in unit of photons/second, $M_{g}$ is the number of photons in the $g^{\text {th }}$ mode group, $\tau$ is the round-trip time within the cavity, $\rho, s$, and $l$ are, respectively, the $\mathrm{Er}^{3+}$ density, the core cross-sectional area, and the length of the EDF, $g_{p}=-\alpha_{p}\left(1-N_{2}\right)$ is the length-averaged pump absorption coefficient, and $g_{g}=\left(\gamma_{g}+\alpha_{g}\right) N_{2}-\alpha_{g}$ is the length-averaged gain coefficient for the $g^{\text {th }}$ mode group, where $\alpha_{p}$ is the pump absorption coefficient and $\gamma_{g}$ and $\alpha_{g}$ are, respectively, the emission coefficient and absorption coefficient of the EDF for the $g^{\text {th }}$ mode group. The four terms on the right-hand-side (RHS) of Eq. (1) correspond to the time-rate changes of $N_{2}$ due, respectively, to the pump excitation, the spontaneous emission, the stimulated emission, and the amplified spontaneous emission (ASE). The time-rate change of $M_{g}$ is given by the following rate equation:

$$
\frac{d M_{g}}{d t}=\frac{1}{\tau}\left(M_{g}\left(G_{f g} e^{g_{g} l-\alpha_{c}}-1\right)+2 m \gamma_{g} l N_{2} \frac{e^{g_{g} l}-1}{g_{g} l}\right)
$$

where $\alpha_{c}$ is the cold-cavity loss (loss external to the EDF) minus the losses from the SFBG and LFBG. The two terms on the RHS of Eq. (2) represent the time-rate change ofphotons due, respectively, to the net gain of a round trip of the laser cavity and the ASE. In Eq. (2), $G_{f g}$ is the parameter denoting the cavity loss contributed by the two FBGs. For simplicity, the reflection spectrum of the LFBG that determines the lasing wavelength is assumed to have a Gaussian shape with a unit peak reflectivity and a half-width at $1 / e$ maximum of $\Delta \lambda$ centered at wavelength of $\lambda_{0}$. Because of the narrow spectral 
TABLE I

Summary of Symbol Definitions and Values of Some Constant PARAMETERS USED IN THIS PAPER

\begin{tabular}{|c|c|c|c|}
\hline Symbol & Value & Unit & Description \\
\hline$\rho$ & $2 \times 10^{24}$ & ions $/ \mathrm{m}^{3}$ & Erbium-ion density \\
\hline$S$ & 8.04 & $\mu \mathrm{m}^{2}$ & Core cross-section area of EDF \\
\hline$l$ & 3 & $\mathrm{~m}$ & Length of EDF \\
\hline$\gamma_{g}$ & 3.7 & $\mathrm{~dB} / \mathrm{m}$ & $\begin{array}{l}\text { Emission coefficient of EDF at } g^{t h} \\
\text { mode group }\end{array}$ \\
\hline$\alpha_{g}$ & 3 & $\mathrm{~dB} / \mathrm{m}$ & $\begin{array}{l}\text { Absorption coefficient of EDF at } g^{\text {th }} \\
\text { mode group }\end{array}$ \\
\hline$\alpha_{p}$ & 4.64 & $\mathrm{~dB} / \mathrm{m}$ & $\begin{array}{l}\text { Pump absorption coefficient at pump } \\
\text { wavelength }\end{array}$ \\
\hline$\Delta \lambda$ & 20 & $\mathrm{pm}$ & $\begin{array}{l}\text { Half-width at } 1 / \mathrm{e} \text { maximum of the } \\
\text { reflection spectrum of the LFBG }\end{array}$ \\
\hline$\lambda_{0}$ & 1550 & $\mathrm{~nm}$ & $\begin{array}{l}\text { Center wavelength of the reflection } \\
\text { spectrum of the long FBG }\end{array}$ \\
\hline$\tau_{0}$ & 10.2 & $\mathrm{~ms}$ & Lifetime of the upper state \\
\hline$\delta \lambda_{m} *$ & 0.1 & $\mathrm{pm}$ & $\begin{array}{l}\text { Maximum wavelength shift induced } \\
\text { by ultrasonic wave }\end{array}$ \\
\hline$k$ & 5 & $\mathrm{~nm}^{-1}$ & $\begin{array}{l}\text { Spectral slope of the SFBG at lasing } \\
\text { wavelength }\end{array}$ \\
\hline$R_{0}$ & 0.8 & & Reflectivity of the SFBG at $\lambda_{0}$ \\
\hline$G$ & 100 & & Number of the total mode groups \\
\hline$m$ & 1 & & $\begin{array}{l}\text { Number of longitudinal modes } \\
\text { within each mode group }\end{array}$ \\
\hline
\end{tabular}

width of the LFBG, the overall loss spectrum resulting from the two FBGs can be assumed to have the same Gaussian shape with the peak reflectivity modulated by the reflectivity of the SFBG at $\lambda_{0}$ and the ultrasonic waves to be detected; then $G_{f g}$ can be expressed as

$$
G_{f g}=e^{-\left(\left(\lambda-\lambda_{0}\right) / \Delta \lambda\right)^{2}}\left(R_{0}+k \delta \lambda_{m} \sin (2 \pi f t)\right)
$$

where $\lambda$ is the wavelength, $R_{0}$ is the reflectivity of the SFBG at $\lambda_{0}, f$ is ultrasonic frequency, $\delta \lambda_{m}$ is the magnitude of the relative wavelength shift of the two FBGs, and $k$ is spectral slope of the SFBG at the lasing wavelength. Finally, the output signal power is proportional to the laser power, $P_{\text {out }}$, which is a summation over all mode groups and given by

$$
P_{\text {out }}=\frac{h c}{\lambda \tau} \sum_{g=1}^{G} M_{g}
$$

where $h$ is the Planck's constant and $c$ is the speed of light in vacuum. A list of the symbols and their definitions and numerical values used in this paper is given in Table I.

Equations (1)-(4) are solved by the $4^{\text {th }}$-order Runge-Kutta method to obtain the static state when no ultrasonic signal is present, which is used as the initial condition to find the temporal response of the FRL to an ultrasonic signal. In this work, the ultrasonic signal is assumed to be a sinusoidal ultrasonic wave with a frequency of $40 \mathrm{kHz}$ and a constant amplitude that leads to a peak relative wavelength shift of $0.1 \mathrm{pm}$ (unless otherwise specified) between the two FBGs, same as used in the experiment described in Section 3. Using the parameters listed in Table I, Fig. 2(a) shows the temporal changes of the FRL output power in response to the ultrasonic waves.
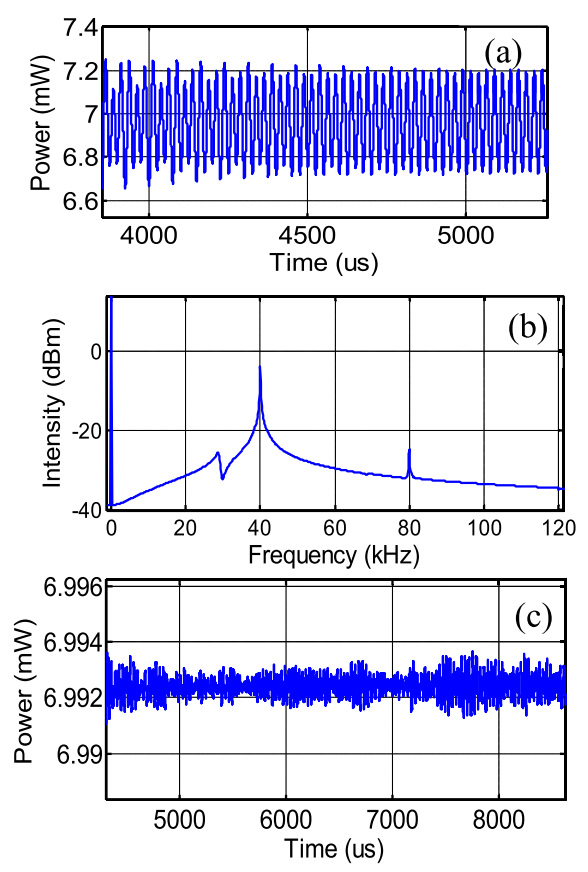

Fig. 2. Calculated temporal response to (a) continuous sinusoidal ultrasonic wave with a frequency of $40 \mathrm{kHz}$ and (b) its FFT spectrum of (a). (c) Calculated temporal response to a Gaussian noise perturbation to the relative wavelength shift of the FBGs.

By carrying out the fast Fourier transform (FFT) of the temporal response, we obtain the frequency response of the system as shown in Fig. 2(b). The first peak is the dc component corresponding to the average power of the laser output. The second peak at $\sim 28 \mathrm{kHz}$ is the RO frequency of the laser cavity, which arises from the transient response induced by the arrival of the ultrasonic signal. The third peak at $40 \mathrm{kHz}$ is the response to the ultrasonic wave, which is the signal peak. The last peak at $80 \mathrm{kHz}$ is believed to be the second-order harmonic generation originating from the nonlinear nature of the laser operation [10].

In order to calculate the SNR of the sensor output, the noise performance of the FRL needs to be analyzed. The FRL noise is simulated by assuming a noise source that randomly varies the relative spectral shifts between the two FBGs. It is mathematically achieved by replacing the sinusoidal function in Eq. (3) with a random function that has a Gaussian distribution with a mean value of 0 and a standard deviation of $5 \times 10^{-3} \mathrm{pm}(1 / 20$ of the peak wavelength shift caused by the ultrasonic signal). A typical simulated temporal noise is shown in Fig. 2(c). To obtain the SNR, we use the peak-to-peak value of the temporal response with ultrasounds as the signal and the root-mean-square value of the temporal response without ultrasound as the noise.

\section{B. Numerical Results}

Figure 3(a) shows the simulation results of the SNR of the sensor system with respect to the pump power when the cold cavity loss is $7.5 \mathrm{~dB}$. It can be seen that the SNR exhibit a non-monotonic dependence on the pump power. The SNR first increases as the pump power increase until 

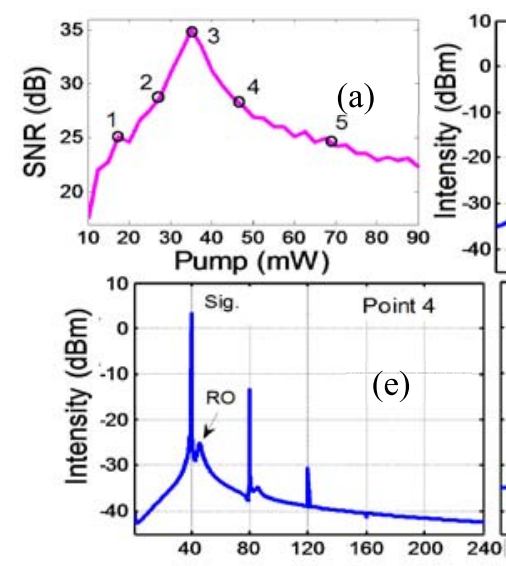
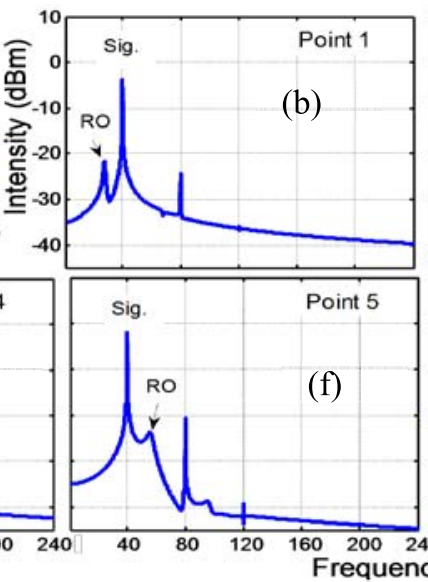
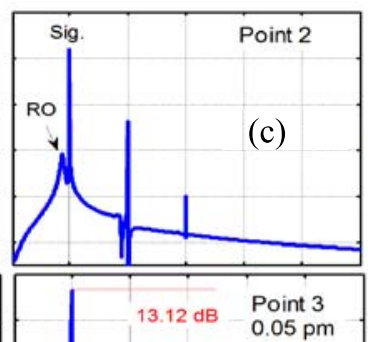

$(\mathrm{g})$

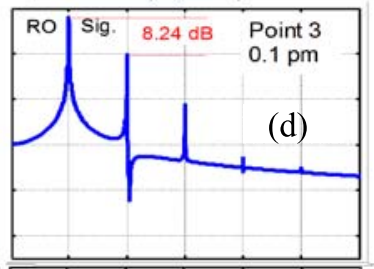

(d)

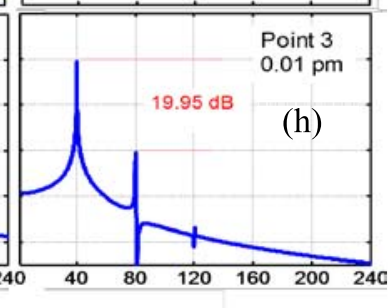

Fig. 3. Simulated (a) SNR of the sensor with respect to pump power and (b)-(f) the FFT spectra corresponding to different operation points 1-5 in (a). The wavelength shift $\left(\delta \lambda_{m}\right)$ is reduced to $(\mathrm{g}) 0.05 \mathrm{pm}$ and $(\mathrm{h}) 0.01 \mathrm{pm}$ when the working condition is kept at point 3 . Sig., signal.

it reaches its peak value [point 3 in Fig. 3(a)]. Then the SNR decreases as the pump power continues to increase. It is revealed that the RO plays a central role in determination of the SNR by examining the spectra of the system output at various operation conditions. Figure 3(b)-(f) shows the spectra of the system output at the operational conditions corresponding, respectively, to points 1-5 in Fig. 3(a). The simulated RO frequencies at points 1-5 are, respectively, $27.6,35.23,40.0,45.7$, and $55.6 \mathrm{kHz}$, indicating that the RO frequency shifts toward higher frequency as pump power increases. Maximum SNR is achieved at point 3 where the RO frequency $(40.0 \mathrm{kHz})$ coincides with that of the ultrasonic signal. Therefore, the sensor reaches maximum SNR when the ultrasound is in resonance with RO of the FRL. It is worth noting that the system output power, which can be obtained by the value of the dc components in Fig. 3(b)-(f), increases monotonically as pump power increases. It is also noted that harmonic generations become more prominent when the ultrasound is in resonance with the RO. For example, in Fig. 3(b), (c), (e), and (f), when the RO frequency is away from the ultrasonic frequency, only the $2^{\text {nd }}$ and $3^{\text {rd }}$ order harmonics may be seen. However, as shown in Fig. 3(d), harmonic peaks up to the $5^{\text {th }}$ order are visible in the spectrum when the ultrasound is in resonance with the RO. In this case, the intensity of the $2^{\text {nd }}$-order harmonic signal is $8.24 \mathrm{~dB}$ lower than that of the signal. Obviously, these harmonic signals are in general undesirable for sensing applications. As aforementioned, we believe these harmonic signals originate from the nonlinear response of the fiber laser. As a simple verification, we calculate the spectral responses of the system to different magnitude of the relative wavelength shifts $\left(\delta \lambda_{m}\right)$ of 0.05 and 0.01 when RO is maintained resonant with the ultrasound and the results are shown in Fig. 3(g) and (h), respectively. In addition to the drop in the signal peak as $\delta \lambda_{m}$ decreases, the intensity difference between the signal peak and the second harmonic peak is increased from $8.24 \mathrm{~dB}$ (Fig. 3(d)) to $19.95 \mathrm{~dB}$ (Fig. 3(h)) when the maximum wavelength shift is decreased from $0.1 \mathrm{pm}$ to $0.01 \mathrm{pm}$. It can be seen that the relative intensities of the harmonic generations are highly dependent on the strength
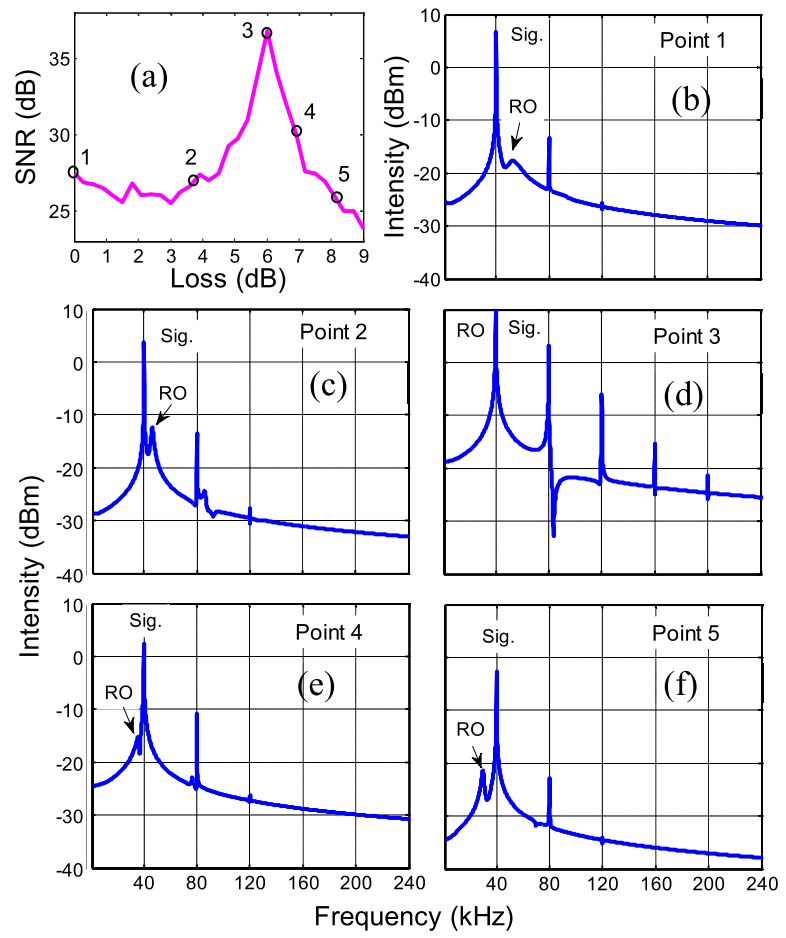

Fig. 4. Simulated (a) SNR of the sensor with respect to cavity loss and (b)-(f) the FFT spectra corresponding to different operation points $1-5$ in (a).

of the ultrasonic signal and harmonic generations are less an issue for low ultrasonic signal strength. However, the harmonic generation around the RO frequency has to be considered for detection of strong and broad-band ultrasonic signals where the output signal from the sensor system may be distorted from the ultrasonic signals due to the presence of strong multiple harmonic components.

Next, we study the SNR of the sensor system with respect to the FRL cold-cavity loss (round-trip loss of the FRL cavity with EDF excluded) when pump power is fixed at $25 \mathrm{~mW}$. The results are shown in Fig. 4. The dependence of the SNR on the cold cavity loss is also non-monotonic and more complicated. When the cold cavity loss is very small $(<3 \mathrm{~dB})$, 

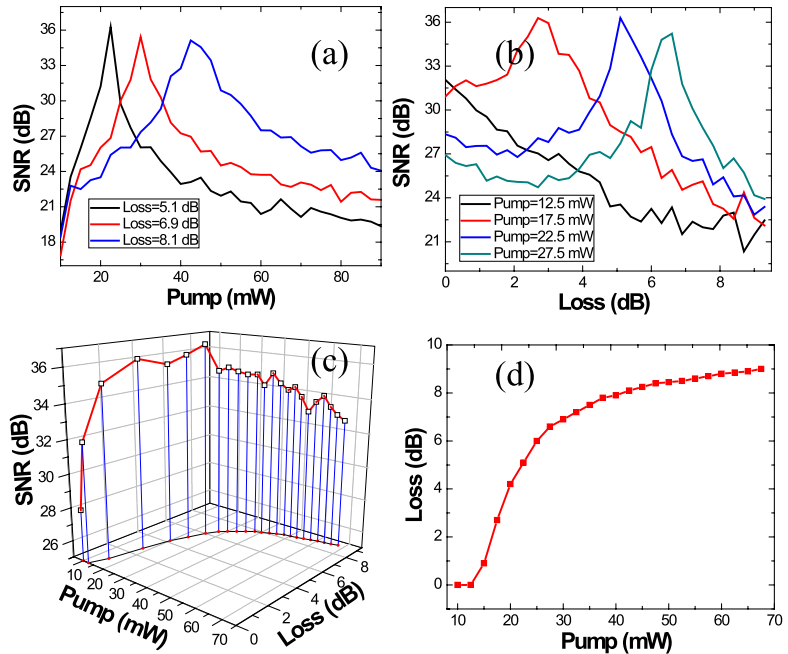

Fig. 5. Simulated output with respect to (a) pump power at different losses and (b) loss at different pump power levels. (c) Output at optimal working points (red) and with no cavity loss (black). (d) Optimal working points (red curve in (c)).

the SNR slightly decreases as the loss increases. Then the SNR starts to exhibit a sharp increase as the loss continues to increase and reaches its peak value when the cold cavity loss is $\sim 6 \mathrm{~dB}$. As the losses continue to increase, the SNR decreases rapidly. The spectra of the system output are calculated for the five operational conditions as indicated by points (1)-(5) in Fig. 4(a) and the results are shown in Fig. 4(b)-(f). The RO frequencies are 52.8, 46.2, 40.3, 35.9, and $29.6 \mathrm{kHz}$ corresponding, respectively, to operation points from 1 to 5 in Fig. 4(a). Again, it is revealed that the peak SNR is reached when the ultrasonic frequency is the same as the RO frequency. Here, the RO frequency as a function of the loss is also nonmonotonic. It is worth noting that, compared to the cases with small $(<3 \mathrm{~dB})$ but non-zero cold-cavity losses, point 1 which denotes a zero cold cavity loss shows slightly increased SNR although its RO frequency is further from the ultrasonic frequency. Similarly, it is seen that higher-order harmonic generations become more prominent as the RO frequency is closer to the ultrasonic frequency. From Fig. 4(d), harmonic peaks up to the $5^{\text {th }}$ order are visible when the ultrasound is in resonance with $\mathrm{RO}$, which is consistent with the results shown in Fig. 3(d).

Our results shown in Figs. 3 and 4 have revealed that the SNR of the FRL sensor system reaches a maximum value when the ultrasound under detection is in resonance with the RO of the FRL. In practical applications, both the pump power and the cold-cavity loss may be tuned within a certain range. It is of great interest to understand how these two parameters can be optimized to achieve the maximum SNR for an ultrasonic signal with a given frequency $(40 \mathrm{kHz}$ in this case). The simulation results shown in Fig. 5 can be used to gain insight into this issue. Fig. 5(a) shows the SNR as a function of the pump power at different cold-cavity losses, suggesting that the pump power to achieve the maximum SNR decreases as cold-cavity loss decreases. Fig. 5(b) is the SNR as a function of cold-cavity loss at different pump powers, indicating that the cold-cavity loss needed to achieve a maximum SNR decreases as the pump power decreases. From these results, we see that, in general, to maintain the $\mathrm{RO}$ frequency at a particular frequency that matches the frequency of the ultrasound under detection for maximum SNR, the cold-cavity cavity loss needs to be increased as the pump power increases. As shown by the black curve in Fig. 5(b), when pump power is too low (e.g. $12.5 \mathrm{~mW}$ in this case), the SNR monotonically decreases as the cold-cavity loss increases from zero, suggesting that the RO frequency cannot be tuned to be in resonance with the ultrasonic frequency. For these cases, we need to minimize the cold-cavity loss to obtain a higher SNR. In order to have a more straightforward and complete view on the effect of the two parameters on the system SNR, we show in Fig. 5(c) the three-dimensional plot of the maximum system SNR as functions of the pump power and the cold-cavity loss. Note that when pump power is less than $12.5 \mathrm{~mW}$, maximum SNR is always achieved when the cold-cavity loss is zero. As the pump power increases further, the cold-cavity loss required to achieve the maximum SNR also increases. The calculated maximum SNR in Fig. 5(c) is $36.82 \mathrm{~dB}$ when the pump power is $25 \mathrm{~mW}$ and the cavity loss is $6 \mathrm{~dB}$. Therefore, the optimal output will decrease if the cavity loss is too large.

Another key factor that affects the practical application of the FRL sensor is its cavity length. Figures 6(a) and (b) are, respectively, the SNR at the ultrasonic frequency of $40 \mathrm{kHz}$ and the RO frequency of the FRL as functions of the FRL cavity length. In Fig. 6(a), a maximum SNR is obtained when the cavity length is around $19 \mathrm{~m}$, which is exactly the point where RO frequency is identical to that of ultrasound $(40 \mathrm{kHz})$, as indicated by the solid dot in Fig. 6(b). From Fig. 6(b), it is seen that the RO frequency monotonically decreases as the cavity length increases. Fig. 6(c)-(e) demonstrates the temporal response to ultrasound for cavity lengths of $28 \mathrm{~m}, 78 \mathrm{~m}$, and $128 \mathrm{~m}$, respectively. It is seen that increased cavity length significantly suppresses its response to the ultrasonic wave. The noise is also suppressed, but to a much lesser degree, leading to a greatly degraded SNR at excessively extended cavity length. This result can be analytically inferred from Eq. (2). The extended cavity length is responsible for an increased round-trip time $\tau$ on the RHS of Eq. (2), resulting in a decreased time-rate change of the lasing photon number $M_{g}$ [left-hand side of Eq. (2)]. Therefore, the applications of FRL ultrasonic sensor system for remote sensing where a long cavity length is required may be limited.

\section{EXPERIMENTS}

Experiments to verify the above theory have been carried out using a setup schematically shown in Fig. 7 . The $\sim 3 \mathrm{~m}$ long EDF (Fibrecore, I-6) was pumped by a $980 \mathrm{~nm}$ diode laser through a 980/1550 nm WDM. The residual pump was removed by another WDM. The cold cavity loss was changed by a tunable attenuator whose attenuation was read through two identical couplers (97/3) placed before and after the attenuator. The two FBGs were mounted side-by-side onto a $2 \mathrm{~mm}$ thick aluminum plate. The power within the laser 

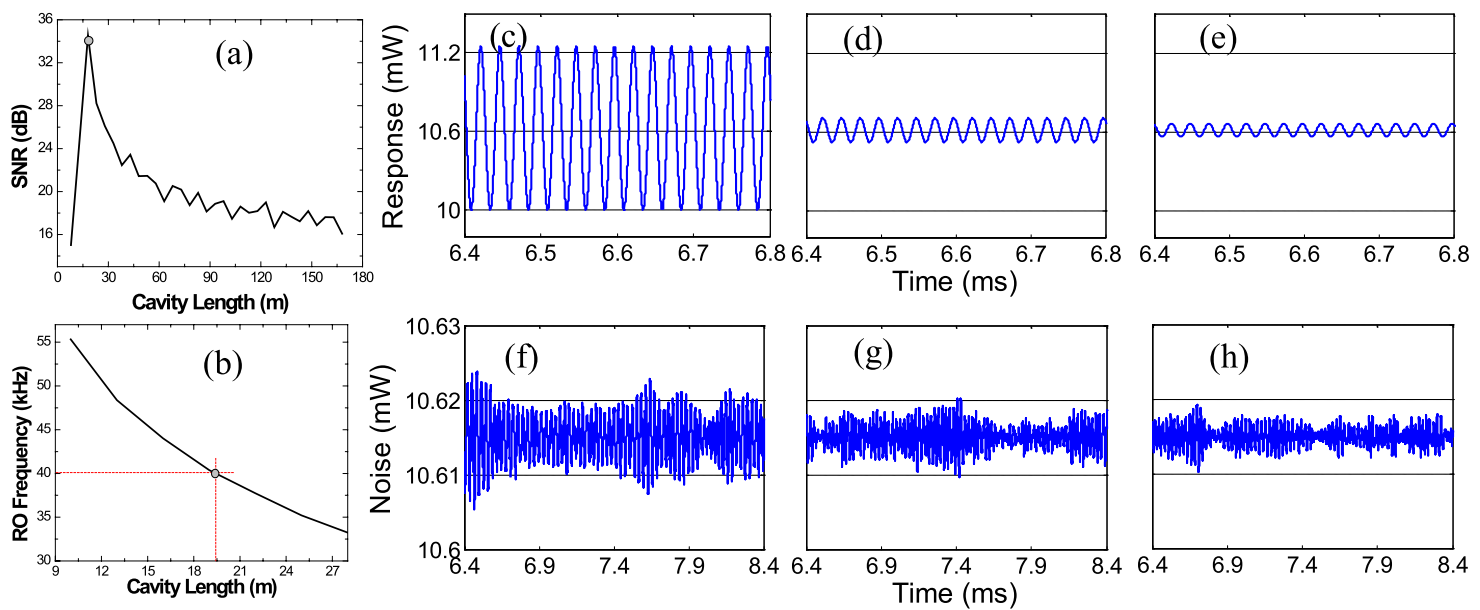

Fig. 6. Calculated (a) SNR versus cavity length and (b) RO frequency versus cavity length around the peak point (denoted by a solid circle) in (a). Simulated output signal and noise when cavity length is (c, f) $28 \mathrm{~m},(\mathrm{~d}, \mathrm{~g}) 78 \mathrm{~m}$, and $(\mathrm{e}, \mathrm{h}) 128 \mathrm{~m}$.

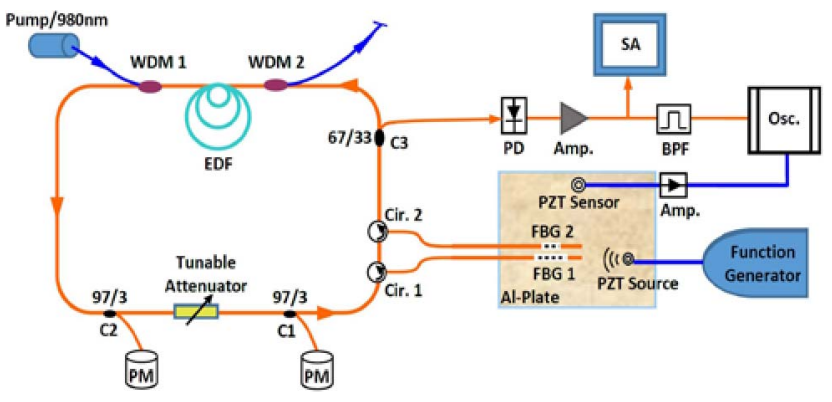

Fig. 7. Experimental setup. PM: power meter; PD: photo detector; Amp.: amplifier; SA: spectrum analyzer; Osc.: oscilloscope. BPF.:bandpass filter.

cavity was tapped out partially through a $67 / 33$ coupler, which was detected by a photodetector and amplified by a broad bandwidth amplifier (20 kHz-1.2 MHz). The amplified signal was sent to an electrical spectrum analyzer (SA) to measure its spectrum. The amplified signal then passed a bandpass filter $(100 \mathrm{~Hz}$ to $300 \mathrm{kHz}$ ) before it was displayed on an oscilloscope. The ultrasound was produced via a commercial piezoelectric transducer driven by a function generator. Another piezoelectric sensor served as a reference. In the absence of ultrasonic waves, the base cold-cavity loss (extra loss with the tunable attenuator excluded) of the system in Fig. 7 was estimated to be about $7.32 \mathrm{~dB}$ which included losses from 11 splicing points $(0.1 \mathrm{~dB}$ each $)$, two $97 / 3$ couplers (0.13 dB each), one 67/33 coupler (1.74 dB), two WDMs (0.13 dB each), two FBGs (3.96 dB in total). The total coldcavity loss was varied by the tunable attenuator. Throughout our experiment, the ultrasonic frequency maintained at $40 \mathrm{kHz}$ same as used in the simulations in Section 2 and the peak-to-peak values of the FRL were used as the signal. The noise was obtained by calculating the root-mean-square value of the FRL sensor system output when the piezoelectric ultrasonic transducer was turned off. During the experiment, the response of the system to ultrasonic signals did not shown any dependence on the polarization state of the light.
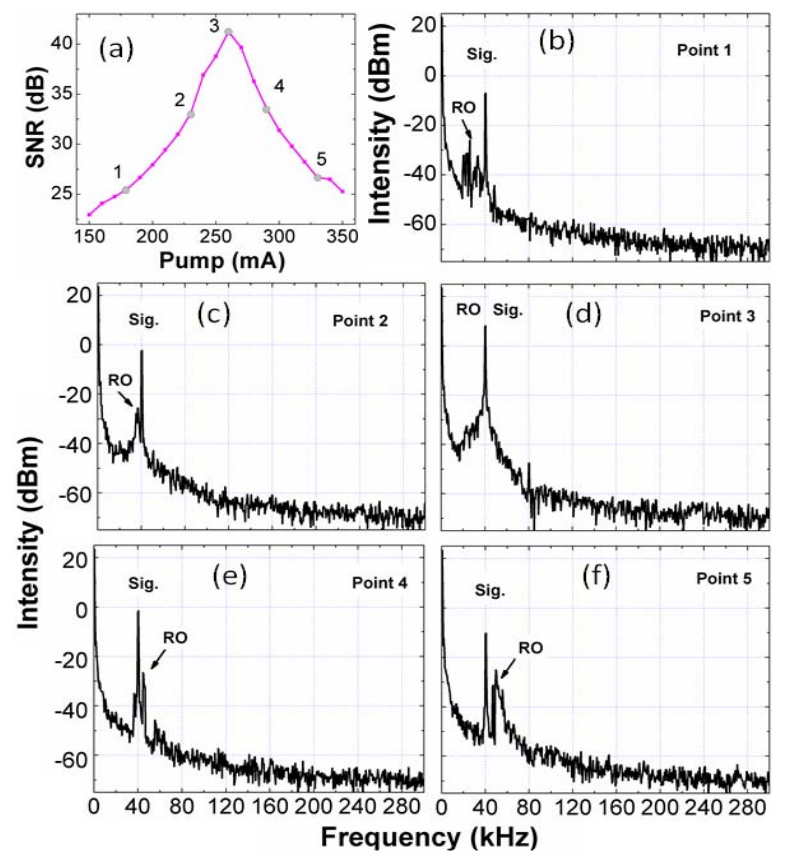

Fig. 8. (a) Experimental SNR of the sensor with respect to pump power and (b)-(f) the FFT curves corresponding to different points 1-5 in (a).

Therefore, polarization controllers that were originally placed inside the FRL cavity were removed.

Figure 8(a) shows the experimental results on the SNR as the pump power (in the unit of pump laser driving current) changes. In this experiment, the total cavity loss was about $8.6 \mathrm{~dB}$. The SNR changed non-monotonically as the pump power increased. The maximum SNR was achieved at the pump input current of $260 \mathrm{~mA}$. Figs. 8(b)-(d) are the output spectra measured by the SA, corresponding, respectively, to the operation points 1 to 5 in Fig. 8(a). Both the RO peak and the signal peak at $40 \mathrm{kHz}$ are evident in all the spectra. The RO frequency increased from $27 \mathrm{kHz}$ to $50 \mathrm{kHz}$ from points 1 to 5 . At point 3 , the RO frequency matches that 

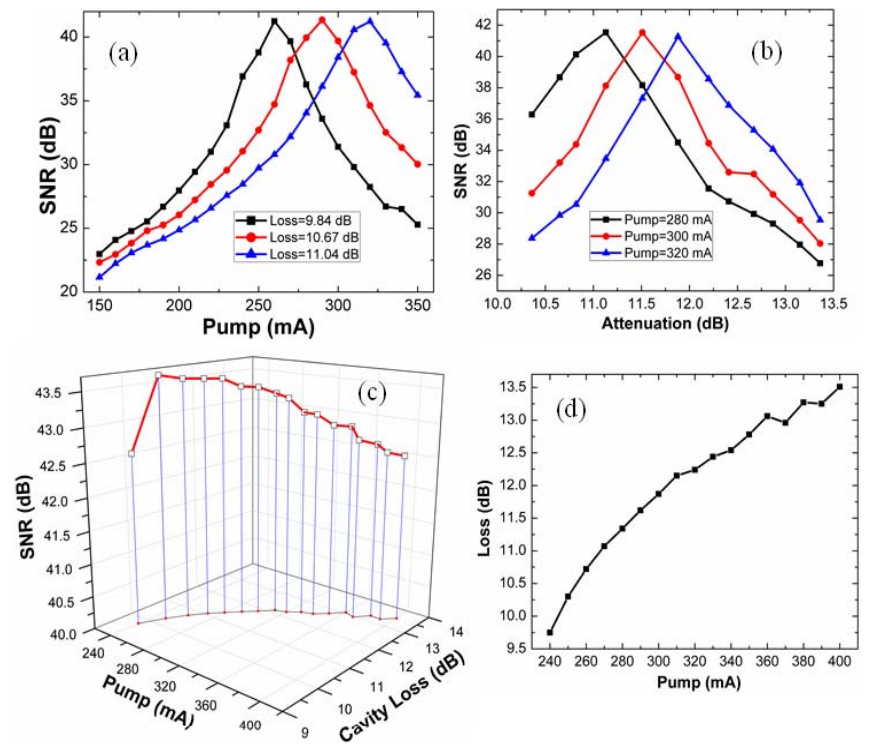

Fig. 9. Experimental SNR with respect to (a) pump at different losses and (b) loss at different pump currents. (c) SNR at optimal working points. (d) Loss-pump pair at optimal working points (projection of the curve on pump and cavity loss plane in (c)).

of the ultrasound, leading to a maximum SNR. Note that when ultrasound was in resonance with RO (point 3), the second harmonic generation was visible and no harmonic components were visible in other spectra for which the RO frequencies were different from the ultrasonic frequency. We note that other higher-order harmonic generations were not observed when the RO and the ultrasonic in resonance, which is believed to be due to the very small ultrasonic signal generated from the piezoelectric transducer (Physical Acoustic Co., Model: HD50) used in the experiment whose resonant frequency $(500 \mathrm{kHz})$ was far larger than the operational frequency $(40 \mathrm{kHz})$, leading to a very low ultrasonic generation efficiency. As predicted by Fig. 3(d) (g) (h), the intensity of the harmonic generations decreases as the ultrasonic signal decrease. The large difference of $55.4 \mathrm{~dB}$ between the signal intensity and the second harmonic intensity may also be an indication that the relative wavelength shift is very small. Except for the higher-order harmonics, the experimental results in Fig. 8(a)-(f) agree well with the theoretical analysis of Fig. 3(a)-(f), respectively.

Figure 9(a) shows the measured SNR as a function of the pump power for different cold-cavity losses, which demonstrates that a larger cold-cavity loss leads to a higher optimal power. The measured SNR as a function of cold-cavity loss at different pump powers is shown in Fig. 9(b), suggesting that a higher optimal power corresponds to a larger coldcavity loss. Due to the base cold-cavity loss $(7.32 \mathrm{~dB})$ of the system, characterization of SNRs at lower cold-cavity loss could not be performed. As a summary, the optimal working points and the according output powers are shown in Fig. 9(c), which shows that the optimal SNR decreases when the loss becomes excessively large. To be clearer, the projection of the three-dimensional curve shown in Fig. 9(c) onto the pump and loss plane is plotted in Fig. 9(d). Obviously, a larger
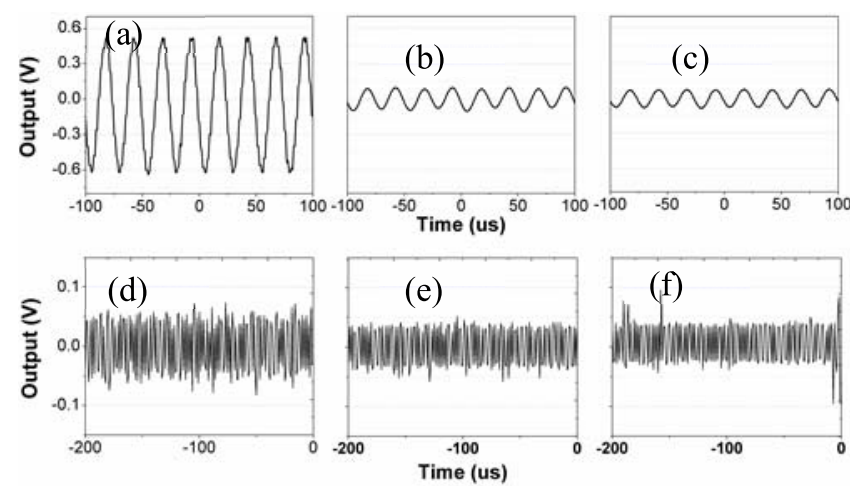

Fig. 10. Experimental output signal and noise when the cavity length is (a, d) $28 \mathrm{~m},(\mathrm{~b}, \mathrm{e}) 78 \mathrm{~m}$, and (c, f) $128 \mathrm{~m}$.

pump power will result in a wider cavity loss range that constitutes the optimal working range. The experimental results in Fig. 9(a)-(d) agree well with the theoretical analysis in Fig. 5(a)-(d), respectively.

Finally, we studied the influence of the FRL cavity length on the system SNR. In this experiment, the cavity length of the original FRL was increased from $28 \mathrm{~m}$ to $78 \mathrm{~m}$ and $128 \mathrm{~m}$ by adding more fiber in the cavity. Figs. 10(a)-(c) and (d)-(e) show, respectively, the system output in response to the $40 \mathrm{kHz}$ ultrasonic wave and the system noise at the three different cavity lengths. It is seen that the peak-to-peak output of the original system with a cavity length of $28 \mathrm{~m}$ [Fig. 10(a)] was reduced by more than 3 times when an extra length of $50 \mathrm{~m}$ fiber [Fig. 10(b)] was added within the cavity. The response was further reduced when the cavity length was increased to $128 \mathrm{~m}$ [Fig. 10(c)], although to a much lesser degree. Figure 10(d)-(f) reveals that the noise was only slightly smaller when the cavity length was increased, suggesting a decrease in SNR since the signal was greatly dropped. These experimental results in general agree with theoretical predictions shown in Fig. 6(c)-(h).

\section{CONCLUSION}

We have studied the SNR of an intensity-demodulated FRL ultrasonic sensor system where the ultrasound-induced relative spectral shifts between a pair of FBGs are demodulated by the laser power variations. We have both theoretically and experimentally analyzed the effect of the key FRL parameters such as the pump power, the cold-cavity loss, and the coldcavity length on the SNR of FRL sensor system output. The experimental results agree well with the theoretical results. Our analysis has revealed that the sensor system exhibits a maximum SNR when the frequency of the ultrasound matches the RO frequency of the FRL. Harmonic generations may be significant for strong ultrasonic signals when the ultrasonic frequency is close to the RO frequency. Therefore, for the detection of strong and broadband ultrasonic signals, tradeoff between the distortion from the harmonic components and sensitivity of the system has to be considered. We have shown that the RO frequency of the FRL can be tuned over a large frequency band by adjusting the pump power and cold-cavity cavity loss to achieve the maximum SNR. 
If the pump power is low, SNR decreases monotonically as the cold-cavity loss increases. When the pump power is high, maximum SNR occurs at a non-zero cold-cavity loss which results in a maximum SNR for an ultrasonic wave at a given frequency. Excessive cold-cavity loss will cause the maximum SNR to decrease dramatically. In addition, the SNR drops exponentially if the cavity length is too large, limiting the applications of the sensor systems to relatively short space range. Finally, it is worth pointing out that, although continuous single frequency ultrasonic waves are considered here for the study of the spectral response of the sensor system, the ultrasonic signal to be detected in practical applications may have a bandwidth much wider than that of the RO peak. In this case, due to the non-flatness of the spectral response of the sensor system, the spectrum of the system output will be different from the spectrum of the ultrasonic signal under detection. However, with the spectral response of the system known, both the spectrum and the time-domain representation of the ultrasonic signal can be recovered from the system output.

\section{REFERENCES}

[1] G. Wild and S. Hinckley, "Acousto-ultrasonic optical fiber sensors: Overview and state-of-the-art," IEEE Sensors J., vol. 8, no. 7, pp. 1184-1183, Jul. 2008.

[2] R. Chen, G. F. Fernando, T. Butler, and R. A. Badcock, "A novel ultrasound fibre optic sensor based on a fused-tapered optical fibre coupler," Meas. Sci. Technol., vol. 15, no. 8, pp. 1490-1495, 2004.

[3] H. Tsutsui, A. Kawamata, T. Sanda, and N. Takeda, "Detection of impact damage of stiffened composite panels using embedded small-diameter optical fibers," Smart Mater. Struct., vol. 13, no. 6, pp. 1284-1290, 2004

[4] N. E. Fisher et al., "In-fibre Bragg gratings for ultrasonic medical applications," Meas. Sci. Technol., vol. 8, no. 10, pp. 1050-1054, 1997.

[5] J. A. Bucaro, H. D. Dardy, and E. F. Carome, "Optical fiber acoustic sensor," Appl. Opt., vol. 16, no. 7, pp. 1761-1762, 1977.

[6] F. Guo, T. Fink, M. Han, L. Koester, J. Turner, and J. Huang, "Highsensitivity, high-frequency extrinsic Fabry-Perot interferometric fibertip sensor based on a thin silver diaphragm," Opt. Lett., vol. 37, no. 9, pp. 1505-1507, May 2012.

[7] P. Lu, Y. Xu, F. Baset, X. Bao, and R. Bhardwaj, "In-line fiber microcantilever vibration sensor," Appl. Phys. Lett., vol. 103, no. 21, pp. 211113-1-211113-5, Nov. 2013.

[8] B. Xu et al., "Acoustic vibration sensor based on nonadiabatic tapered fibers," Opt. Lett., vol. 37, no. 22, pp. 4768-4770, Nov. 2012.

[9] H. Tsuda, "Bragg wavelength-insensitive fiber Bragg grating ultrasound detection system based on a fiber ring laser," Proc. SPIE, vol. 7753, p. 77538J, May 2011.

[10] M. Han, T. Liu, L. Hu, and Q. Zhang, "Intensity-demodulated fiber-ring laser sensor system for acoustic emission detection," Opt. Exp., vol. 21, no. 24, pp. 29269-29276, Dec. 2013

[11] Q. Wu, Y. Okabe, and J. Sun, "Investigation of dynamic properties of erbium fiber laser for ultrasonic sensing," Opt. Exp., vol. 22, no. 7, pp. 8405-8419, Apr. 2014.

[12] T. Liu, L. Hu, and M. Han, "Adaptive ultrasonic sensor using a fiber ring laser with tandem fiber Bragg gratings," Opt. Lett., vol. 39, no. 15, pp. 4462-4465, Aug. 2014
[13] M. A. Mirza and G. Stewart, "Multiwavelength operation of erbium-doped fiber lasers by periodic filtering and phase modulation," J. Lightw. Technol., vol. 27, no. 8, pp. 1034-1044, Apr. 15, 2009.

[14] G. Stewart, G. Whitenett, K. Vijayraghavan, and S. Sridaran, "Investigation of the dynamic response of erbium fiber lasers with potential application for sensors," J. Lightw. Technol., vol. 25, no. 7, pp. 1786-1796, Jul. 2007.

[15] Y. Sun, J. L. Zyskind, and A. K. Srivastava, "Average inversion level, modeling, and physics of erbium-doped fiber amplifiers," IEEE J. Sel. Topics Quantum Electron., vol. 3, no. 4, pp. 991-1007, Aug. 1997.

Guigen Liu received the B.E. degree in mechanical engineering from Qingdao University, Qingdao, China, in 2008, and the Ph.D. degree in mechanical engineering from the Changchun Institute of Optics, Fine Mechanics and Physics (CIOMP), Chinese Academy of Sciences, Changchun, China, in 2013. During his Ph.D. phase, his research focused on tapered optical fibers for label-free biochemical detection. After receiving the Ph.D. degree, he was with CIOMP as a Research Assistant. In 2014, he joined the Department of Electrical and Computer Engineering, University of Nebraska-Lincoln, Lincoln, NE, USA, as a Post-Doctoral Research Associate. His current research includes in fiber-optic ultrasound sensors and high-speed temperature sensors.

Elliot Sandfort is currently an Undergraduate Researcher with the Department of Computer Engineering, University of Nebraska-Lincoln, Lincoln, NE, USA, where he was a Summer Intern with the Department of Electrical and Computer Engineering, in 2014.

Lingling Hu was born in Dalian, China, in 1988. She received the B.S degree in physics from the Dalian University of Technology, Dalian, in 2011. Since 2012, she has been a Research Assistant with the Department of Electrical and Computer Engineering, University of Nebraska-Lincoln, Lincoln, NE, USA. Her research interests include optical fiber sensors and fiber lasers.

Tongqing Liu, photograph and biography not available at the time of publication.

Ming Han received the B.S. and M.S. degrees in electronics engineering from Tsinghua University, Beijing, China, in 1998 and 2000, respectively, and the $\mathrm{Ph} . \mathrm{D}$. degree in electrical engineering from the Virginia Polytechnic Institute and State University, Blacksburg, VA, USA, in 2006. He is currently an Associate Professor with the Department of Electrical and Computer Engineering, University of Nebraska-Lincoln, Lincoln, NE, USA. His research interests are in fiber optic sensors, fiber lasers, and optical instrumentation. 